\title{
Forward- and Backward-Second Harmonic Generation Imaging of Corneal and Scleral Collagens
}

\author{
Wen Lo ${ }^{1}$, Hsin-Yuan Tan ${ }^{2,3}$, Ming-Guo Lin ${ }^{1}$, Chu-Mei Hsueh ${ }^{1}$, Wei-Liang Chen ${ }^{1}$, Sun-Jan Lin ${ }^{3,4}$, \\ Shiou-Hwa Jee ${ }^{3,4}$, Chen-Yuan Dong ${ }^{1 *}$ \\ ${ }^{1}$ Department of Physics, National Taiwan University, Taipei 106, Taiwan \\ ${ }^{2}$ Department of Ophthalmology, Chang Gung Memorial Hospital, Linko 333, Taiwan \\ ${ }^{3}$ Institute of Medical Engineering, College of Medicine and College of Engineering, Taipei 100, \\ Taiwan \\ ${ }^{4}$ Department of Dermatology, National Taiwan University Hospital, Taipei 100, Taiwan
}

\section{INTRODUCTION}

Collagen is the most abundant protein in mammalian and forms various types of tissues. On ocular surface, sclera, limbus and cornea are composed with fibril form collagen. However, unlike other connective tissues with high opacity, cornea has extraordinary high transparency which originates from the regular arrangement of collagen fibers within cornea. Cornea is responsible for $80 \%$ of focusing power of our vision and any corneal damage can cause severe vision loss. The high transparency of cornea makes it difficult to probe it without invasive processes, especially stromal structure alternations.

Collagen, however, is an effective second harmonic generator due to its non-centrosymmetric molecule structure and can be visualized with nonlinear optical process without labeling. In addition, the deeper penetration and point like effective volume of SHG can also provide 3-dimensional information with minimum invasion. Backward SHG imaging has been approved effectively demonstrating structure alternation in infective keratitis, thermal damage in cornea, corneal scar, post refractive surgery wound healing and keratoconus which is also a main complication after refractive surgery[1-6]. In practical, backward SHG has the potentiality to be developed as clinical examination modality.

However, Han et al also demonstrated that backward SHG (BSHG) imaging provides collagen bundle information while forward SHG (FSHG) provides more detailed, submicron fibril structure visualization within corneal stroma[7]. In sclera, which also has type I collagen as its main composition, BSHG and FSHG imaging reveal similar morphology. Comparing with what Legare et al demonstrated that BSHG in bulk tissue mainly originate from backscattered FSHG[8], the huge difference between corneal BSHG and FSHG imaging originate from the high transparency of cornea. However, only BSHG could be applied in practical. Therefore, if the correlation of BSHG and FSHG, which reveals more architecture details, can be established, BSHG may be used in clinical examination in the future.

\section{Multiphoton Microscope}

\section{MATERIAL AND METHODS}

In this work, a homebuilt multiphoton microscope, based on a laser scanning system and a commercial microscope (E800; Nikon ${ }^{\mathrm{TM}}$, Japan), is used for imaging purpose. The output of a titanium-sapphire pulse laser (Tsunami ${ }^{\mathrm{TM}}$, Spectra Physics, Mountain View, CA) pumped by a diode-pumped, solid state laser (Millennia ${ }^{\mathrm{TM}} \mathrm{X}$, Spectra Physics, Mountain View, CA) operating at the wavelength of $780 \mathrm{~nm}$ was used as the excitation source. The corresponding SHG wavelength is then centered at $390 \mathrm{~nm}$. Circularly polarized laser is focused by a long working distance water immersion objective (SFluor, WI, 40X, N.A. 0.8, Nikon) onto the sample.

In the backward detecting part, the collected signals first pass through the short-pass, dichroic mirror used in reflecting the excitation source into the focusing objective (700dcspruv-3p, Chroma Technology, Rockingham, VT). The broadband MF and SHG signal are separated by a secondary dichroic mirror (435 dcxr, Chroma Technology) and respectively filtered by two band-pass filters (MF: E435lp-700sp, SHG: HQ390/20, Chroma Technology) before reaching the detectors. Photon-counting photomultiplier tubes (R7400P, Hamamatsu, Japan) were used in the backward detecting system.

Multiphoton Microscopy in the Biomedical Sciences VIII, edited by Ammasi Periasamy, Peter T. C. So, Proc. of SPIE Vol. 6860, 68600F, (2008) · 1605-7422/08/\$18 - doi: 10.1117/12.763437 
In the forward detecting unit, forward SHG signal is collected by a lens then reflected by a dichroic mirror (435 dcxr, Chroma Technology) and filtered by a band-pass filter (HQ390/20). A different photon-counting photomultiplier (R5700P, Hamamatsu, Japan) is used in the forward detecting system.

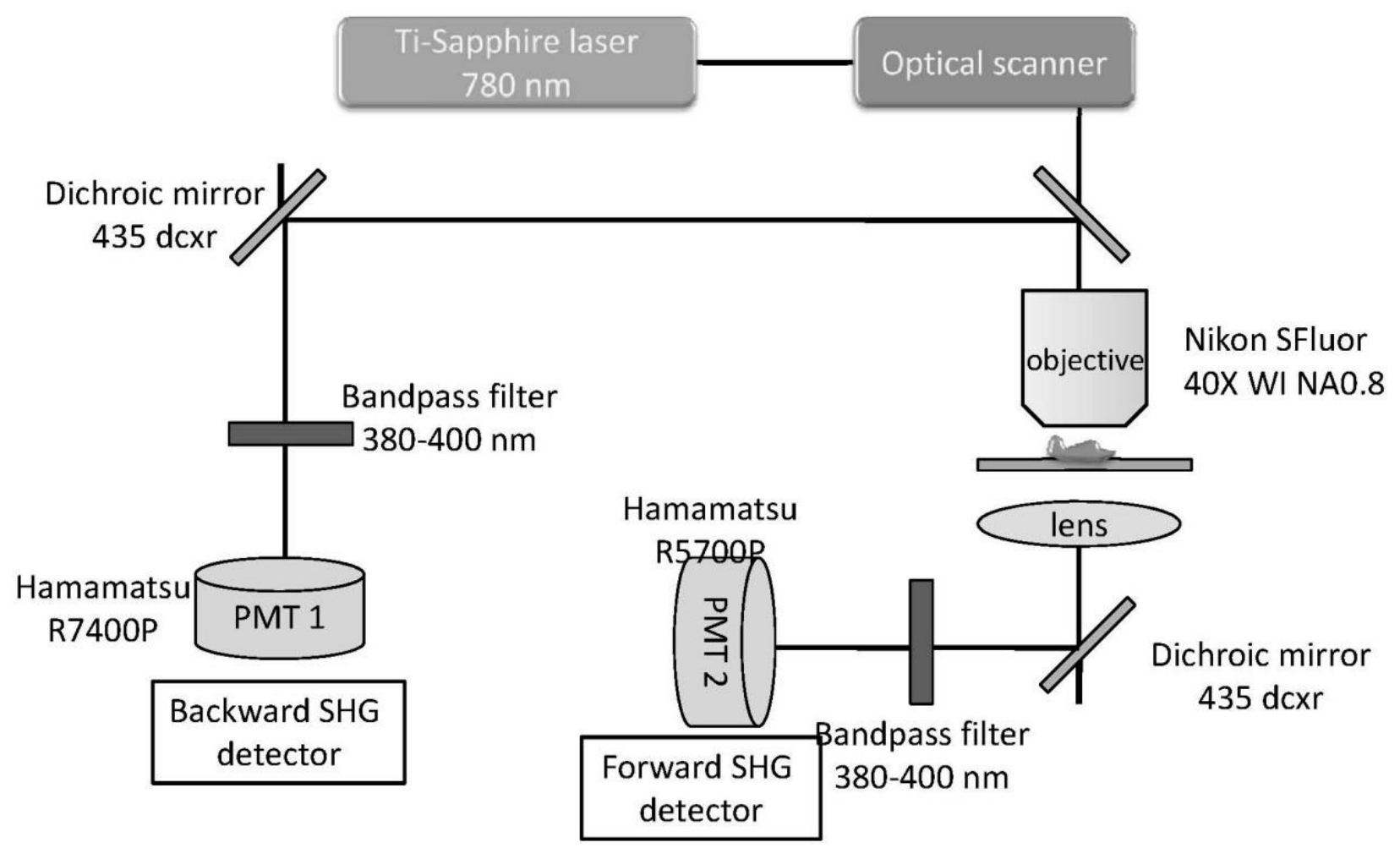

Fig.1 the instrument setup diagram.

\section{Cornea}

To demonstrate how FSHG/BSHG correlation varies in sclera, health cornea and diseased corneas, normal cornealscleral ring, human corneal scar, human corneal edema. Normal human corneal-scleral ring and corneal pathology specimens are obtained from Chang-Gung Memorial Hospital. The study protocol was approved by an institutional review board, and was kept to the Helsinki Declaration with respect to human subjects in biomedical research.

Analysis

From the images we obtained we notice that in some specimens, especially sclera and diseased corneas, FSHG and BSHG images are similar. However, in healthy corneas, BSHG imaging looks like the complementary imaging of FSHG. To quantify this observation, Pearson's linear correlation analysis is used in this work.

\section{RESULTS}

In the cornea region in corneal-scleral ring, two different size scale images are analyzed. In the larger scale analysis, images along the depth with 1 micron depth interval and a 110 by 110 micron frame size are performed intensity profile and correlation analysis. In the smaller scale analysis, 5 regions with size 8 by 8 microns are randomly selected within the larger scale imaging are performed the same analysis. The analyzed results are shown in Fig. 2 and 3 respectively. In Fig 2 small to medium positive correlation between FSHG and BSHG imaging are observed in the larger area analysis. However, in the small area analysis, the correlation doesn't exist. The correlation is higher in the anterior stroma and decreases in deeper stroma.

To demonstrate the image correlation difference between clear cornea and highly opaque sclera, we performed the same analysis along the direction from sclera to cornea. A $2.5 \mathrm{~mm}$ by $2.5 \mathrm{~mm}$ large area image, which covers a part of sclera, limbus and a part of cornea (Fig. 4), is separated in to 25 strip-like regions orthogonal to the direction from sclera to 
cornea for analysis. To obtain statistic results, each strip image is separated into 5 smaller areas and the averaged result is shown in Fig. 5. In Fig. 5, higher FSHG in cornea and higher BSHG in sclera are observed. In addition, the FSHG/BSHG correlation is higher in sclera and limbus. The FSHG/BSHG imaging of corneal scar is shown in Fig. 6. The image is separated into 16 areas $(4 \times 4)$ for analysis. In the scar peripheral the correlation is higher than the scar center. If we observe the image very carefully, we can notice that thicker and more irregularly arranged collagen networks can be found in the scar peripheral. However, in corneal edema specimen, we cannot tell the difference from normal cornea form the morphology. We also cannot find difference in the FSHG/BSHG correlation analysis either.

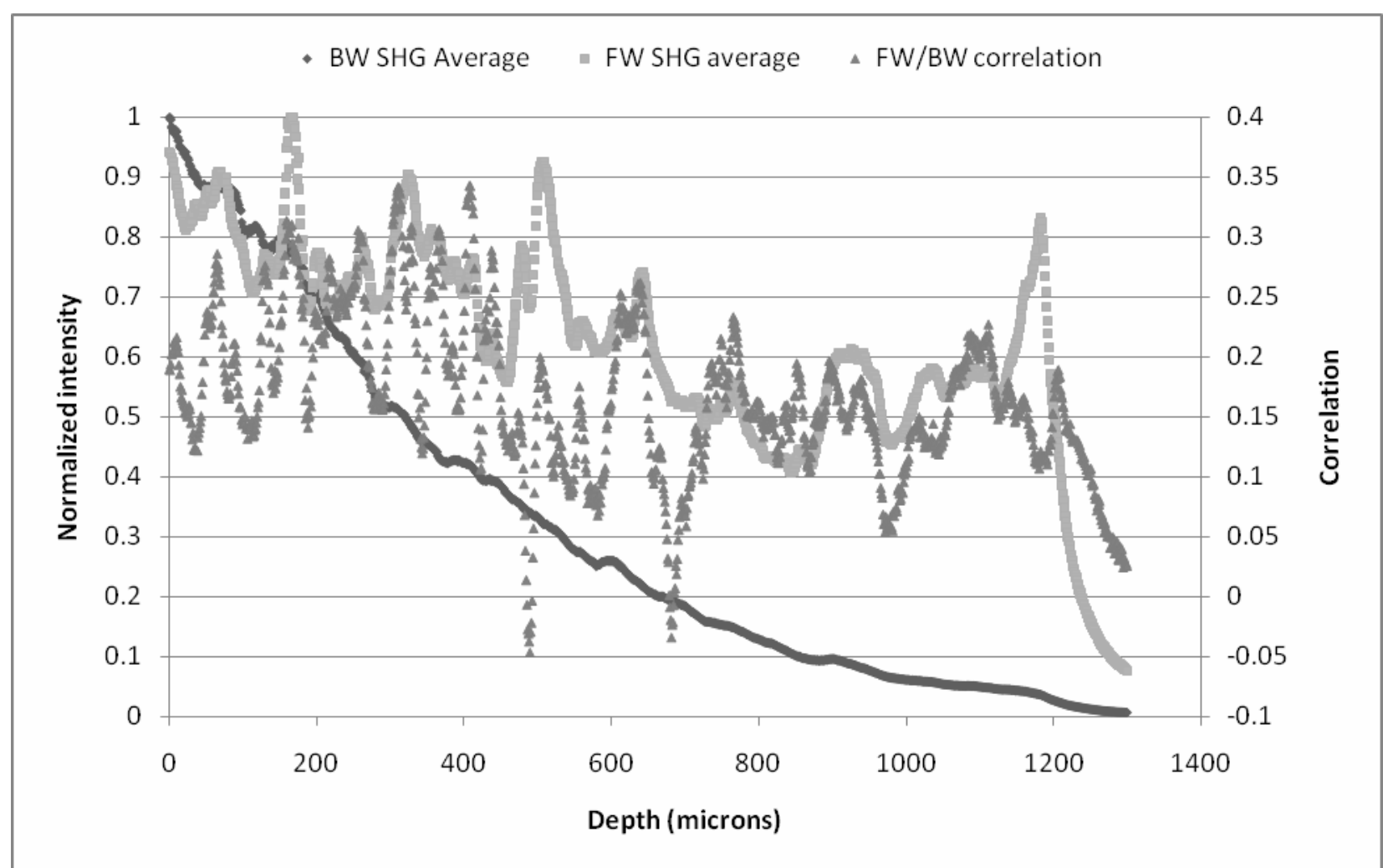

Fig. 2 Quantitatively analysis of FSHG/BSHG intensity profile and their correlation in a lager area (110 by 110 microns). Small to medium correlation can be found and the correlation is higher in the anterior stroma. In addition, the FSHG intensity doesn't drop to half of the highest intensity through entire thickness of cornea while the BSHG drops to half at $1 / 4$ thickness of the corneal button. 


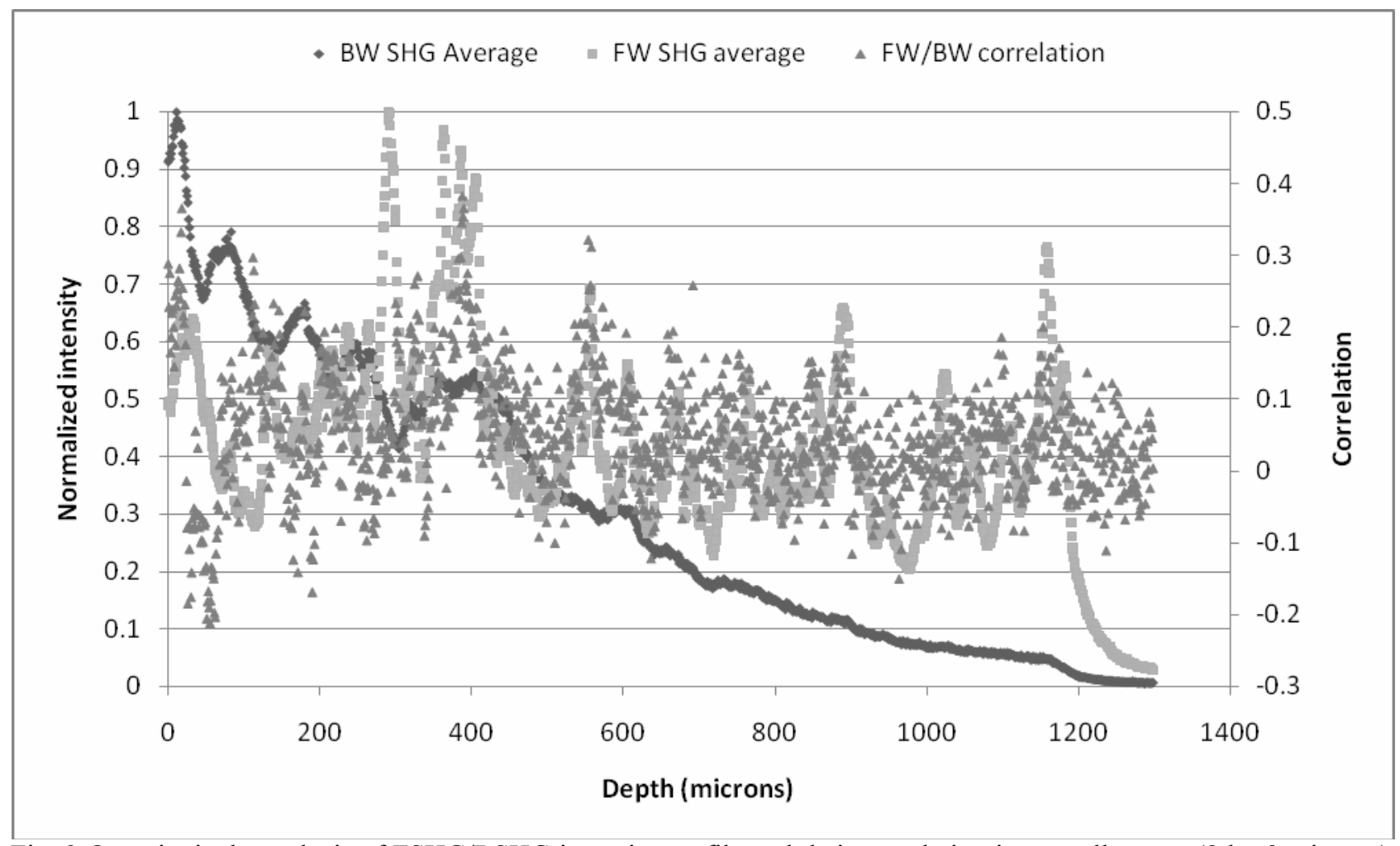

Fig. 3 Quantitatively analysis of FSHG/BSHG intensity profile and their correlation in a smaller area (8 by 8 microns). There is almost no correlation between FSHG and BSHG.
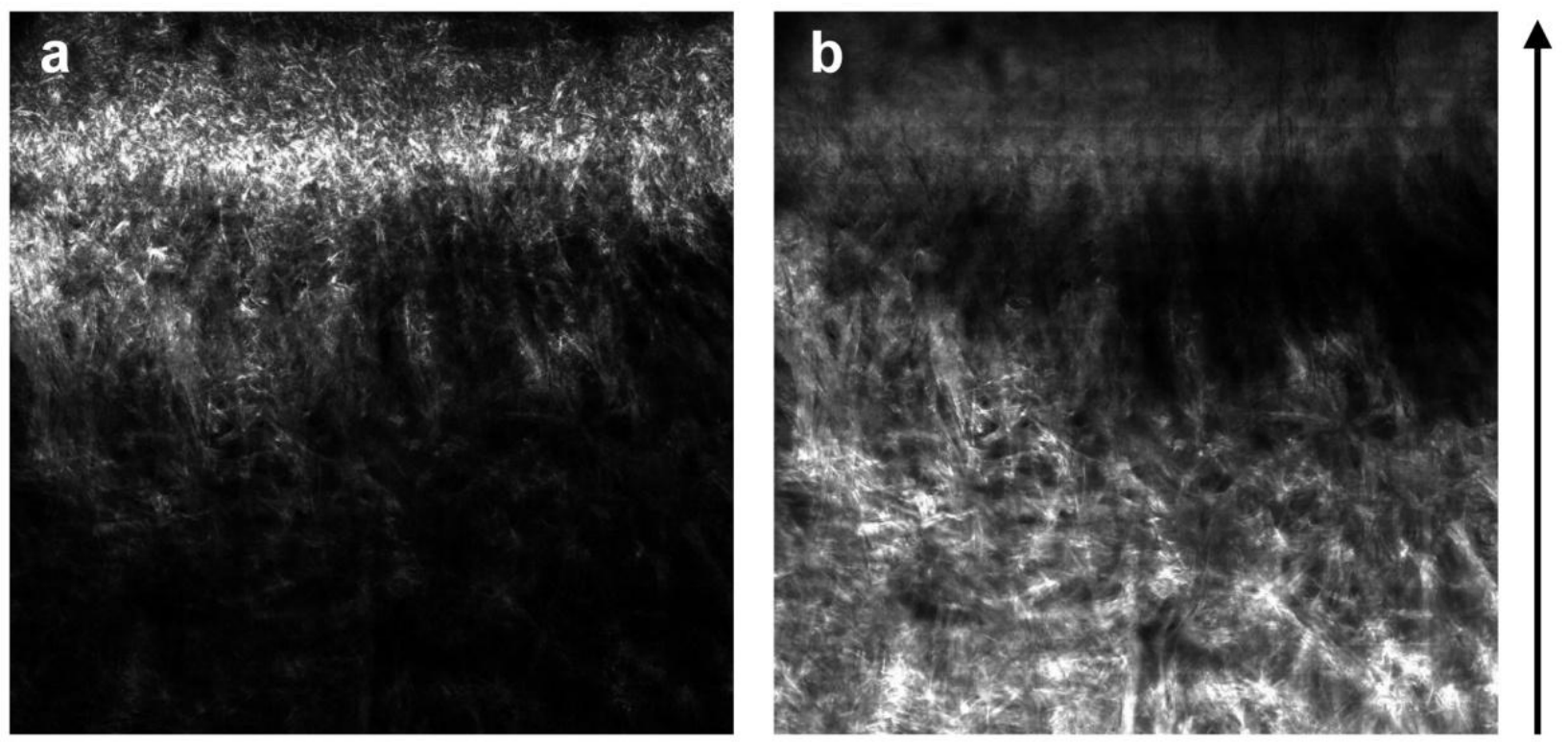
Fig. $4 \mathrm{a}$ and $4 \mathrm{~b}$ are FSHG and BSHG images of normal corneal-scleral ring respectively. The intensity profile and correlation analysis are performed along the arrow indicated direction. From the morphology shown in $4 \mathrm{~b}$, we can recognize that the bottom part of the image is the sclera and the top part covers cornea region.

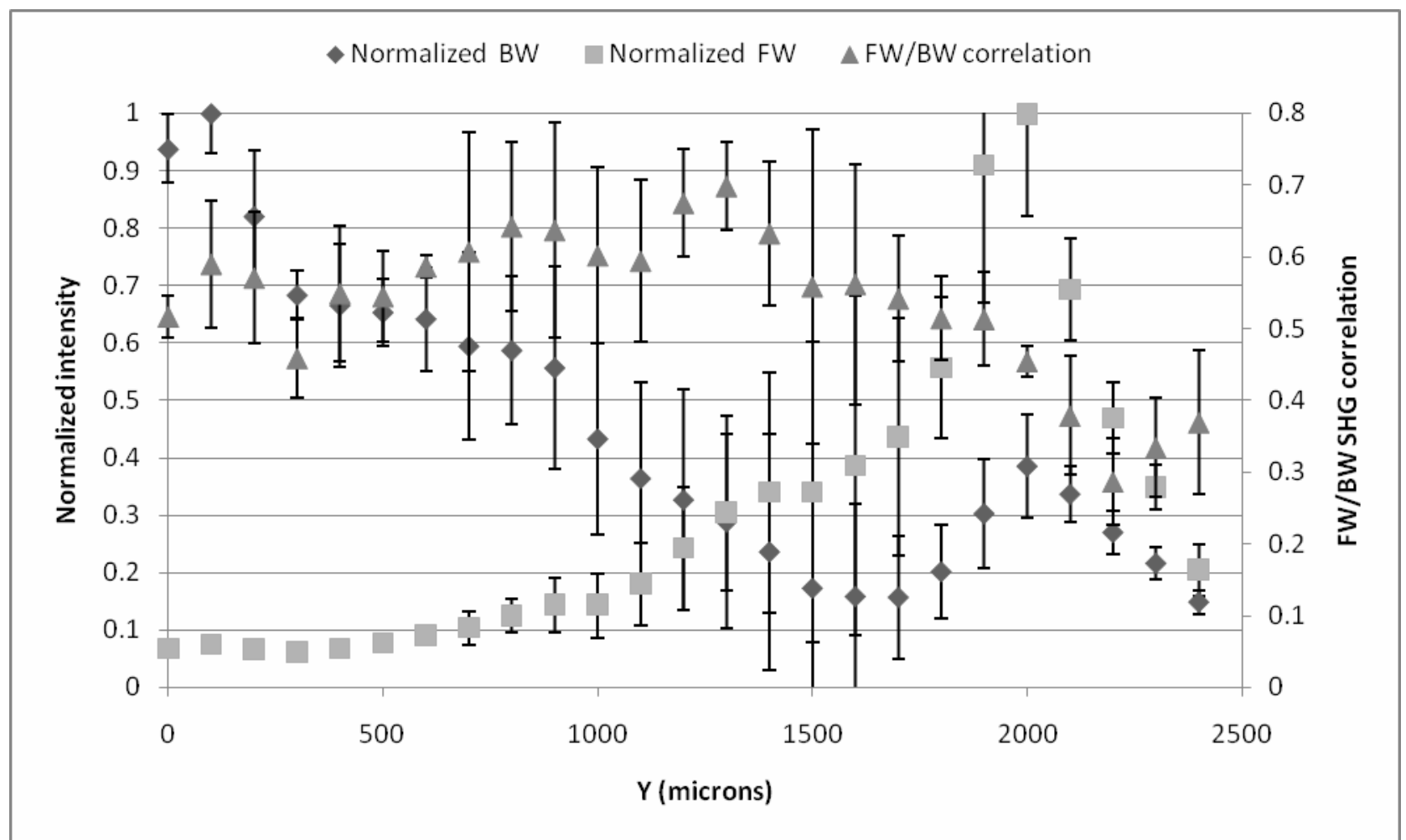

Fig. 5 the intensity profile of forward SHG, backward SHG and FSHG/BSHG correlation are analyzed along the direction from sclera to cornea as the arrow indicates in Fig. 4.
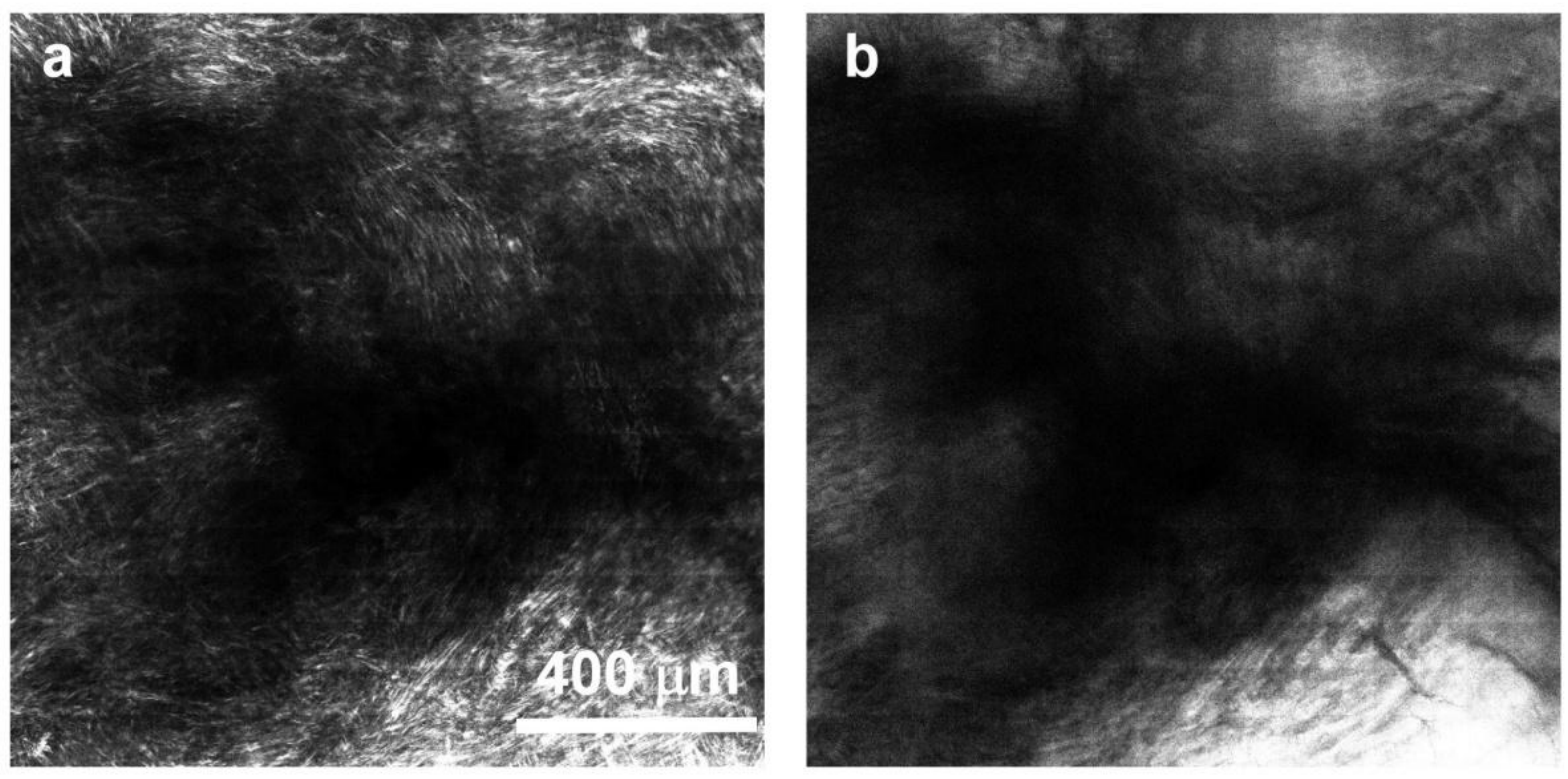
Fig. 6 corneal scar, at the scar peripheral, thicker and more randomized collagen fibers are observed. This image is later averagely separated into a $4 \times 4$ array (16 small areas.) The FSHG/BSHG intensity and correlation analysis is also perform on these small areas and the results are shown in Fig. 7

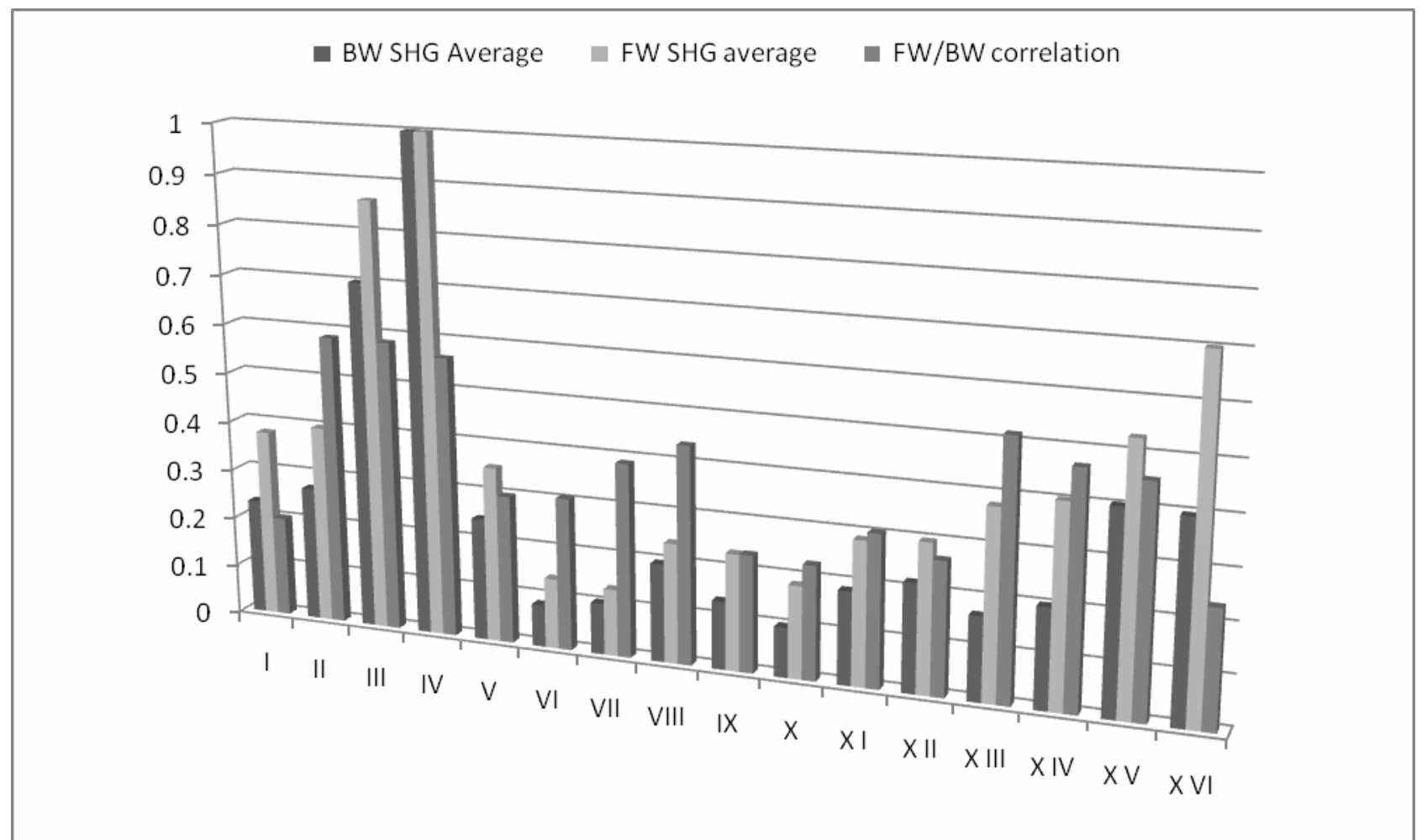

Fig. 7 FSHG/BSHG intensity and correlation analysis of corneal scar. In the scar center (region IX, X, XI), the FSHG/BSHG correlation is comparatively low. In addition due to the absence of thick collagen the FSHG/BSHG intensity are also decreased.

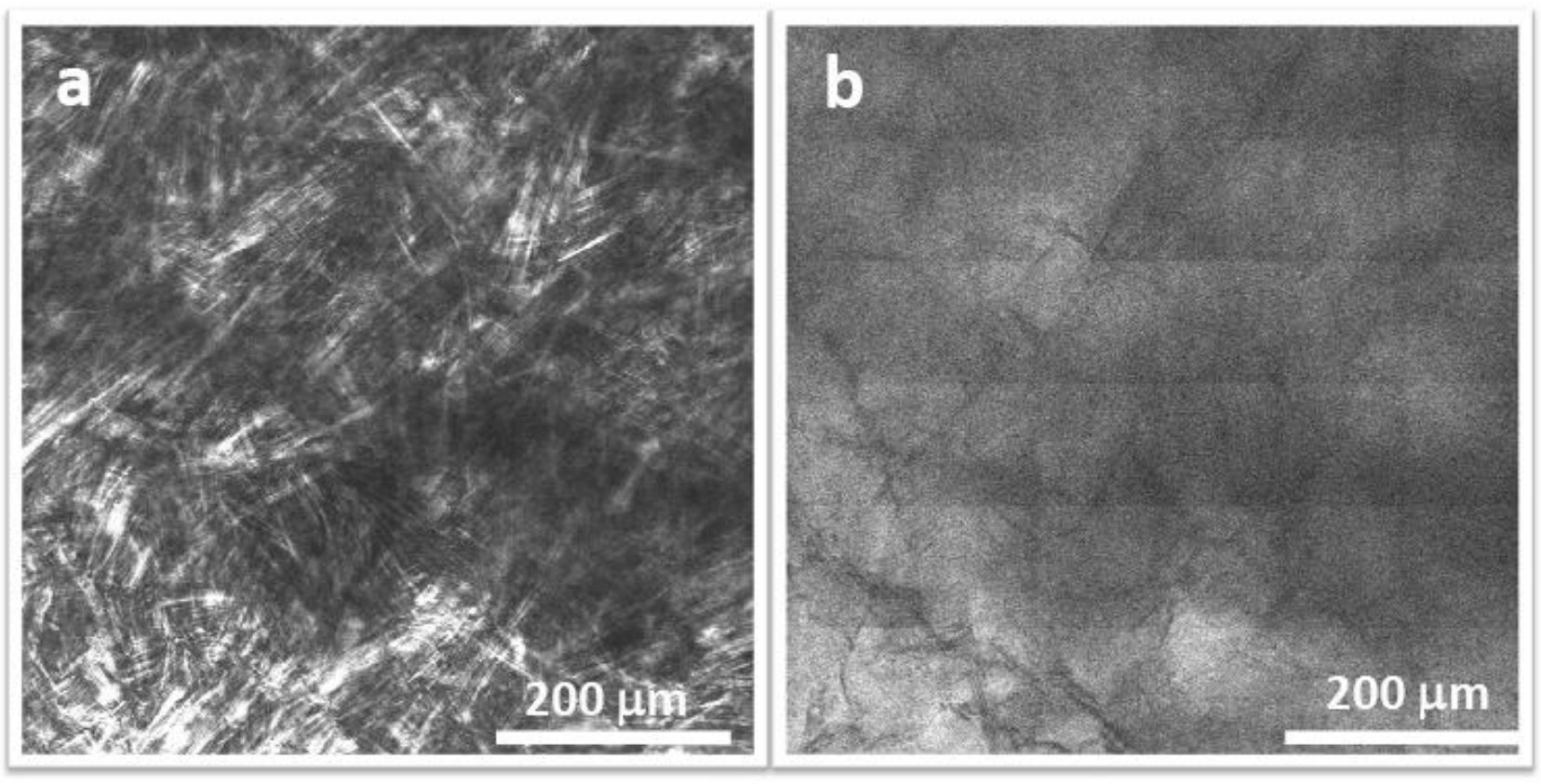


Fig 8a and $\mathrm{b}$ are FSHG and BSHG images of corneal edema respectively. Comparing to normal cornea imaging shown in the cornea part in Fig. 3, there is no significant difference.

\section{CONCLUSION}

These results imply two important points. First, BSHG provides larger scaled bundle information rather than detail fibril structure of collagen in corneal stroma. In addition, in the tissues with less organized collagen fibrils have higher FSHG/BSHG correlation due to the back scattered FSHG. In addition, the BSHG intensity profile decrease fast in both healthy and diseased corneas while the FSHG only decreases fast in the diseased cornea. These results also suggest that BSHG still provides structure information especially in diseased corneas. However, in corneal edema the correlation analysis doesn't show difference comparing to normal corneas even though the opacity obviously increased in corneal edema specimen.

\section{REFERENCES}

[1] H. Y. Tan, S. W. Teng, W. Lo, W. C. Lin, S. J. Lin, S. H. Jee, and C. Y. Dong, "Characterizing the thermally induced structural changes to intact porcine eye, part 1: second harmonic generation imaging of cornea stroma," Journal of Biomedical Optics, vol. 10, pp. -, Sep-Oct 2005.

[2] W. Lo, S. W. Teng, H. Y. Tan, K. H. Kim, H. C. Chen, H. S. Lee, Y. F. Chen, P. T. C. So, and C. Y. Dong, "Intact corneal stroma visualization of GFP mouse revealed by multiphoton imaging," Microscopy Research and Technique, vol. 69, pp. 973-975, Dec 2006.

[3] H. Y. Tan, Y. Sun, W. Lo, S. J. Lin, C. H. Hsiao, Y. F. Chen, S. C. M. Huang, W. C. Lin, S. H. Jee, H. S. Yu, and C. Y. Dong, "Multiphoton fluorescence and second harmonic generation imaging of the structural alterations in keratoconus ex vivo," Investigative Ophthalmology \& Visual Science, vol. 47, pp. 5251-5259, Dec 2006.

[4] S. W. Teng, H. Y. Tan, J. L. Peng, H. H. Lin, K. H. Kim, W. Lo, Y. Sun, W. C. Lin, S. J. Lin, S. H. Jee, P. T. C. So, and C. Y. Dong, "Multiphoton autofluorescence and second-harmonic generation imaging of the ex vivo porcine eye," Investigative Ophthalmology \& Visual Science, vol. 47, pp. 1216-1224, Mar 2006.

[5] H. Y. Tan, Y. Sun, W. Lo, S. W. Teng, R. J. Wu, S. H. Jee, W. C. Lin, C. H. Hsiao, H. C. Lin, Y. F. Chen, D. H. K. Ma, S. C. M. Huang, S. J. Lin, and C. Y. Dong, "Multiphoton fluorescence and second harmonic generation microscopy for imaging infectious keratitis," Journal of Biomedical Optics, vol. 12, pp. -, Mar-Apr 2007.

[6] S. W. Teng, H. Y. Tan, Y. Sun, S. J. Lin, W. Lo, C. M. Hsueh, C. H. Hsiao, W. C. Lin, S. C. M. Huang, and C. Y. Dong, "Multiphoton fluorescence and second-harmonic-generation microscopy for imaging structural alterations in corneal scar tissue in penetrating full-thickness wound," Archives of Ophthalmology, vol. 125, pp. 977-978, Jul 2007.

[7] M. Han, G. Giese, and J. F. Bille, "Second harmonic generation imaging of collagen fibrils in cornea and sclera," Optics Express, vol. 13, pp. 5791-5797, Jul 252005.

[8] F. Legare, C. Pfeffer, and B. R. Olsen, "The role of backscattering in SHG tissue imaging," Biophys J, vol. 93, pp. 1312-20, Aug 152007. 